\title{
Performance of a deep learning CNN model for the \\ automated detection of 13 common conditions on Chest $X$-rays
}

Tirupathi Karthik ${ }^{1}$, Kasiraman Vijayalakshmi ${ }^{1}$, Paski Bavani ${ }^{1}$, Gurram Kashyap ${ }^{1}$, Talwar Amit ${ }^{1}$, Donkena Monisha ${ }^{1}$

Background and aims: Chest X-rays are widely used, non-invasive, cost effective imaging tests. However, the complexity of interpretation and global shortage of radiologists have led to reporting backlogs, delayed diagnosis and a compromised quality of care. A fully automated, reliable artificial intelligence system that can quickly triage abnormal images for urgent radiologist review would be invaluable in the clinical setting. The aim was to develop and validate a deep learning Convoluted Neural Network algorithm to automate the detection of 13 common abnormalities found on Chest X-rays.

Method: In this retrospective study, a VGG 16 deep learning model was trained on images from the Chestray 14 , a large publicly available Chest $X$-ray dataset, containing over 112,120 images with annotations. Images were split into training, validation and testing sets and trained to identify 13 specific abnormalities. The primary performance measures were accuracy and precision.

Results: The model demonstrated an overall accuracy of $88 \%$ in the identification of abnormal X-rays and $87 \%$ in the detection of 13 common chest conditions with no model bias.

Conclusion: This study demonstrates that a well-trained deep learning algorithm can accurately identify multiple abnormalities on the images. As such models get further refined, they can be used to ease radiology workflow bottlenecks and improve reporting efficiency. Napier Healthcare's team that developed this model consists of medical IT professionals who specialise in Al and its practical application in acute \& long-term care settings. This is currently being piloted in a few Hospitals and Diagnostic Labs on a commercial basis.

K. Tirupathi is with Napier Healthcare Technologies Private Limited, \# 04-03, 100, Pasir Panjang Road, Singapore 118518 (email tirupathi.karthik@napierhealthcare.com)

K. Vijayalakshmi is with Napier Healthcare Technologies Private Limited, \# 04-03, 100, Pasir Panjang Road, Singapore 118518 (email k.vijayalakshmi@napierhealthcare.com)

B. Paski is with Napier Healthcare Technologies Private Limited, 1st Floor, Western Aqua, Hi-Tech City, Whitefields,

Kondapur, Hyderabad-500081 (email bhavani@napierhealthcare.com)

K. Gurram is with Napier Healthcare Technologies Private Limited, 1st Floor, Western Aqua, Hi-Tech City, Whitefields,

Kondapur, Hyderabad-500081 (email kashyap.gurram@napierhealthcare.com)

A.Talwar is with Napier Healthcare Technologies Private Limited, 1st Floor, Western Aqua, Hi-Tech City, Whitefields,

Kondapur, Hyderabad-500081 (email amit.talwar@napierhealthcare.com)

M. Donkena is with Napier Healthcare Technologies Private Limited, 1st Floor, Western Aqua, Hi-Tech City, Whitefields,

Kondapur, Hyderabad-500081 (email donkena.monisha@napierhealthcare.com) 


\section{Introduction}

Artificial intelligence $(\mathrm{Al})$ and deep learning are disruptive technologies that have moved at an unimaginable pace from being a futuristic promise to a current reality. The rise and dissemination of $\mathrm{Al}$ and $\mathrm{ML}$ (Machine Learning) have begun to permeate all spheres of our lives and are beginning to be applied to the field of healthcare as well.

In parallel, over the last two decades, advancement in medical imaging technology has led to the exponential growth of the use of diagnostic imaging for the early detection, diagnosis, and treatment of diseases. Imaging has taken on a critical role in modern healthcare, and most patient care pathways are reliant on an efficient radiology service to deliver the best outcomes [1], [2].

Among the various imaging modalities in use, Chest $X$-rays are the most common, and millions of studies are performed globally every year [3], [4], [5]. The wide availability, low cost, non-invasive nature, portability and ease of operation make it an attractive initial choice for the detection of a large number of thoracic conditions [3], [4].

Chest X-ray interpretation, however, is a complex task that is time consuming and labour intensive. Specialist radiologists who are qualified to perform this task are in short supply globally. It is estimated that there are only about 10.83 radiologists for every 100,000 people in the United States, 6.9 per 100,000 people in Canada, around 5 for 100,000 people in the UK and 1 per 100,000 people in India [1]. In Singapore, statistics in 2019 showed that there were only around 392 registered diagnostic radiologists serving a population of around 5.7 million people [6].

In many parts of world, the number of digital X-ray machines available far exceed the availability of professionals to interpret and report on them. Such gaps in the radiology workforce result in backlogs, delayed diagnosis, fatigue based diagnostic errors and poor quality of patient care.

In addition, despite their widespread use, Chest $X$-rays are low resolution images that are not easy to read. The overlapping of the tissue structures in the chest greatly increases the complexity of interpretation, as does the patient position during the study, the exposure technique, and image quality [7]. Significant subjectivity and inter-reader variability depending on the level of expertise and the abnormality being detected, are other factors that add to the complexity [4], [8], [9], [10]. All of these factors have thus resulted in a renewed interest in harnessing the power of $\mathrm{Al}$ and deep learning to assist and augment interpretation of Chest X-ray images by radiologists . A clinically validated, automated, artificial intelligence system that can independently read Chest Xrays could provide substantial benefits such as prioritisation of the workload, clinical decision support to minimise diagnostic errors, large-scale screening and global population health initiatives especially in low resource settings [3], [4], [11], [12]. 
Computer-aided detection (CAD) is not new to radiology. Early attempts at computerised analysis of medical images date back to the 1960s. But the very limited computational power and lack of high-quality digitized image data at that time meant these applications were not very successful [13], [14], [15].

The second era of artificial intelligence through the 1980s and 90s once again saw the widespread use of CAD tools based on 'conventional' machine learning to assist radiologists in image interpretation. The first CAD commercial system was approved by the Food and Drug Administration (FDA) for use in screening mammography in 1998 [16], [17]. However, none of these systems reached the high performance or diagnostic accuracy that offered significant benefits to radiologists.

Over the last few years, there has been increasing interest in the use of deep learning algorithms based on Convoluted Neural Networks (CNNs) to assist with abnormality detection on medical images [18], [19], [20].

The major limitations of conventional ML techniques were the complex feature engineering, significant domain knowledge, and data processing expertise that were required to extract the essential discriminative features to train non-deep learning models. Deep learning systems on the other hand, are able to automate the feature extraction and classification steps, effectively shifting the burden of feature engineering from the human to the machine side [13], [20], [21]. A single well-designed and well-trained network can yield state-of-the-art results across many domains by the use of 'transfer learning' without the need for significant domain knowledge.

A good example is the application of CNNs to image detection in the real-world setting. In the early editions of the ImageNet Large-Scale Visual Recognition Challenge (ILSVRC), the traditional CAD methods produced five times as many errors as a practiced human when attempting to identify everyday objects in photographs [22], [23]. However, in 2012, the convoluted network model 'AlexNet' significantly outperformed other conventional methods and in 2015, the winning CNN algorithm, 'ResNet', exhibited astounding results surpassing human level performance, paving the way for the use of these technologies in other domains as well [24], [25], [26].

'Transfer learning' from ImageNet, has now become a de-facto method for deep learning applications in different fields of medical imaging and several research groups have very successfully applied these models to detect specific problems on Chest X-ray [27], [28]. 


\section{Napier CNN model for Chest X-rays}

In our study, we analysed a supervised multi-label classification framework based on the VGG16 CNN model (ConvNet/CNN), for the automated detection of abnormal images and the identification of 13 common chest conditions on Chest $X$-rays.

\section{OBJECTIVE}

To evaluate and validate the performance of the Napier CNN algorithm for the automated detection of one or more of 13 abnormal radiological findings on Chest X-rays.

\section{DATA}

The Napier algorithm was trained on the ChestX-ray14 data set [29], one of the largest public open-source repositories of chest radiographs released by the National Institute of Health (NIH), that contains 112,120 frontal view images of more than 30,000 unique patients.

\section{METHOD}

28,250 images were selected from the original data set and were randomly split in the ratio of $80: 15: 5$ for training, validation and testing. Thus, the model had a training set of 21,800 images (3150 normal/ 18,650 abnormal), a validation set of 3750 images (500 normal/ 3250 abnormal) and a testing set 2700 images (350 normal/ 2350 abnormal). The class distribution of the conditions in the abnormal set are shown in Table 1 and the adjusted frequency of representation of each condition in shown in Figure 1.

\begin{tabular}{|l|l|}
\hline \multicolumn{1}{|c|}{ Condition } & \multicolumn{1}{c|}{ Number of images } \\
\hline Atelectasis & 3845 \\
\hline Cardiomegaly & 994 \\
\hline Consolidation & 1741 \\
\hline Edema & 497 \\
\hline Effusion & 3970 \\
\hline Emphysema & 726 \\
\hline Fibrosis & 721 \\
\hline Infiltration & 5548 \\
\hline Mass & 1632 \\
\hline Nodule & 2003 \\
\hline Pleural Thickening & 1418 \\
\hline Pneumonia & 386 \\
\hline Pneumothorax & 1606 \\
\hline
\end{tabular}

Table1: The class distribution of the conditions in the abnormal set. 


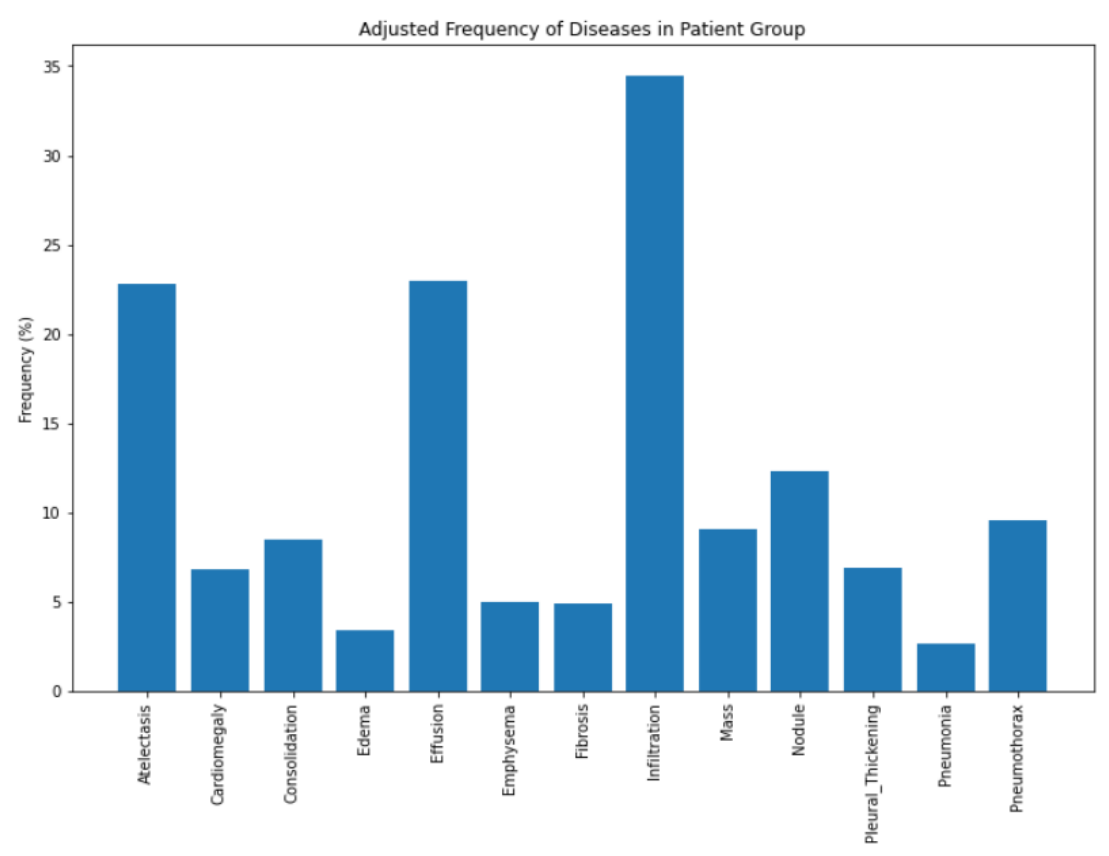

Figure 1: Adjusted frequency of the 13 conditions in our data set

\section{MODEL DEVELOPMENT}

A deep learning 16-layer convolutional neural network was developed. The model takes in an input Chest X-ray image, assigns importance (through learned weights and biases) and delivers an output based on the presence or absence of one or more of the 13 conditions that it has been trained to detect.

\section{IMAGE PROCESSING TECHNIQUES}

The Chest $X$-rays in the dataset varied considerably in size, resolution and quality. The images were resized to a standard size of $224 \times 224$ pixels, and a set of image normalization techniques was applied to reduce variation. Additional data augmentation techniques like the angle tilt were applied before the images were presented to the model.

\section{PRETRAINING}

Pretraining was done on the ImageNet pre-trained weights. During the design and training phase, some selected layers of the CNN were trained, and the respective layer weights were updated based on our use case specific training images.

\section{TRAINING}

Model training was done with 50 + epochs on around 28,000 images.. The number of epochs is a hyperparameter that defines the number of times the learning algorithm will run through the entire training dataset to update the model weights. 


\section{PERFORMANCE ASSESSMENT}

The primary performance metric measures were accuracy, precision, recall and F1 score.

RESULTS

(1) The deep learning model demonstrated an overall accuracy of $88 \%$ in the identification of abnormal X-rays and $87 \%$ in the detection of 13 common chest conditions.

The classification report on sample test data set for normal and abnormal classification of the model is shown in figure 2 .

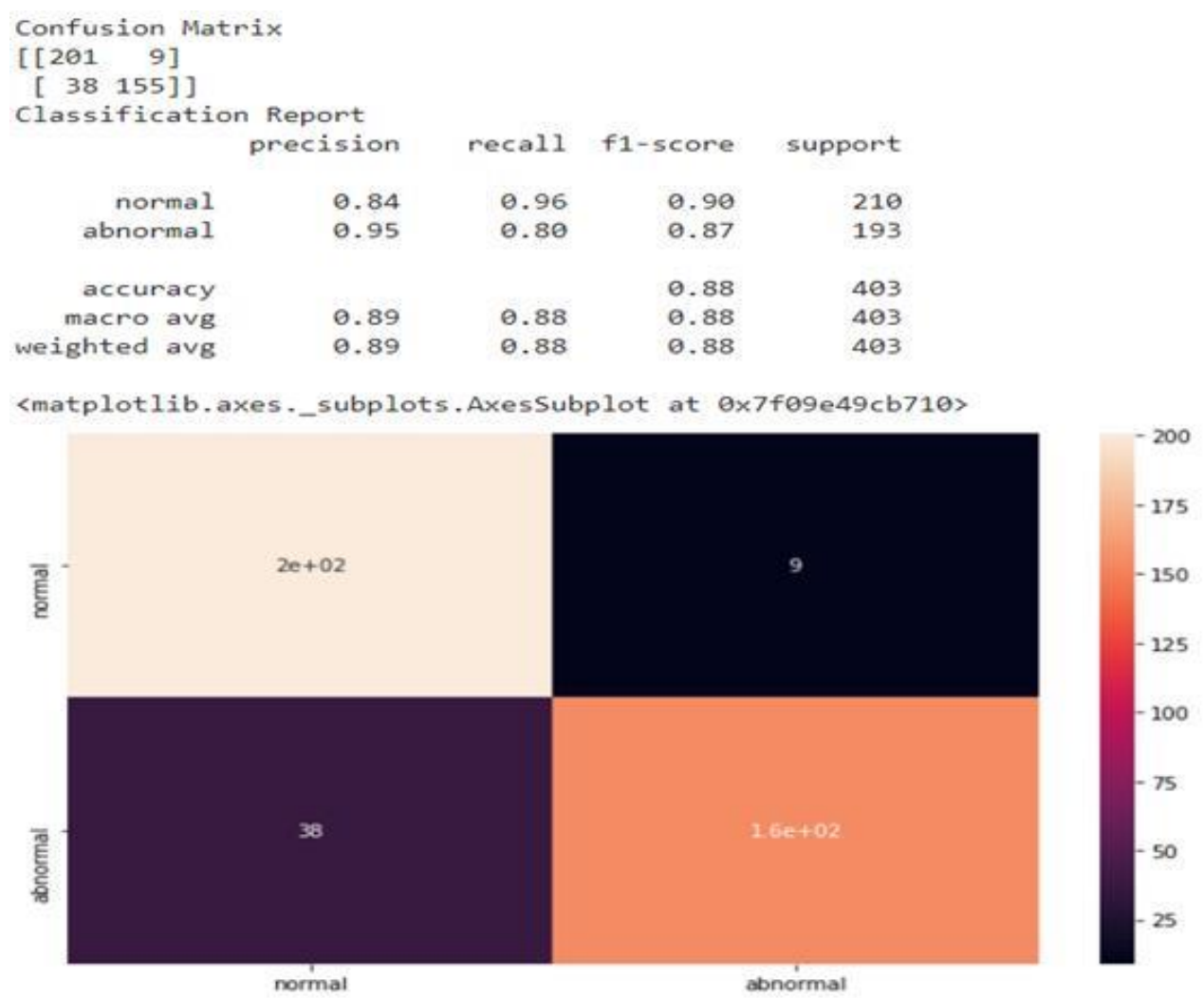

Figure 2: Classification report on sample test data set for normal and abnormal classification of the model

(2) Analysis of accuracy indicated that there was no model bias due to over fitting or variance due to under fitting. Model showed good generalisation over test data as shown in Figure 3.

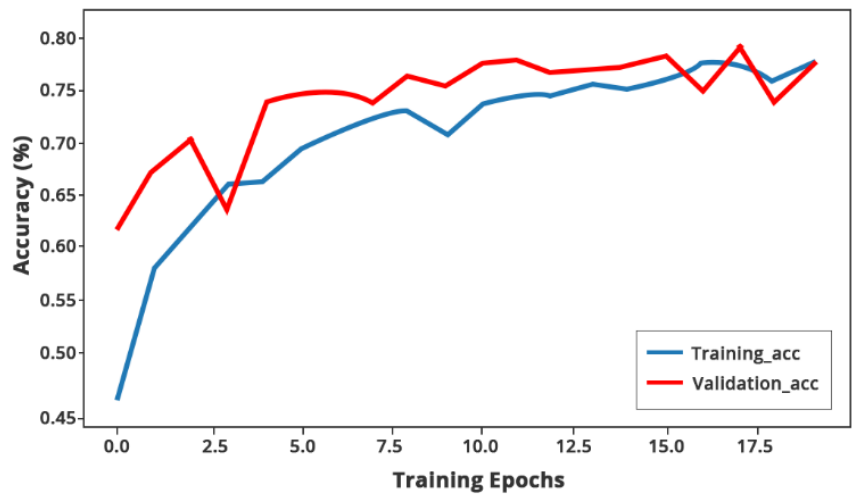

Figure 3: Training vs Validation Accuracy Metric 
(3) The loss curve indicated stable descent of error rate and the model was trained until it reached the optimal loss value, indicated by the curve flattening over time and reaching the optimal threshold value as demonstrated in Figure 4.

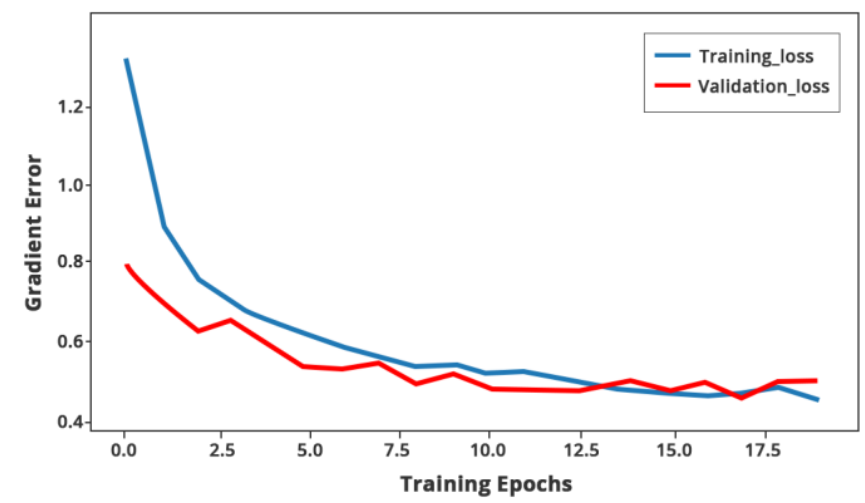

Fig 4: Training vs Validation Loss Curve

(4) The model was able to provide insight into its working in the form of location heatmaps (Gradcam) that identified the ROI (Region of Interest) having higher weightage, or importance in the resultant analysis as displayed in Image 1

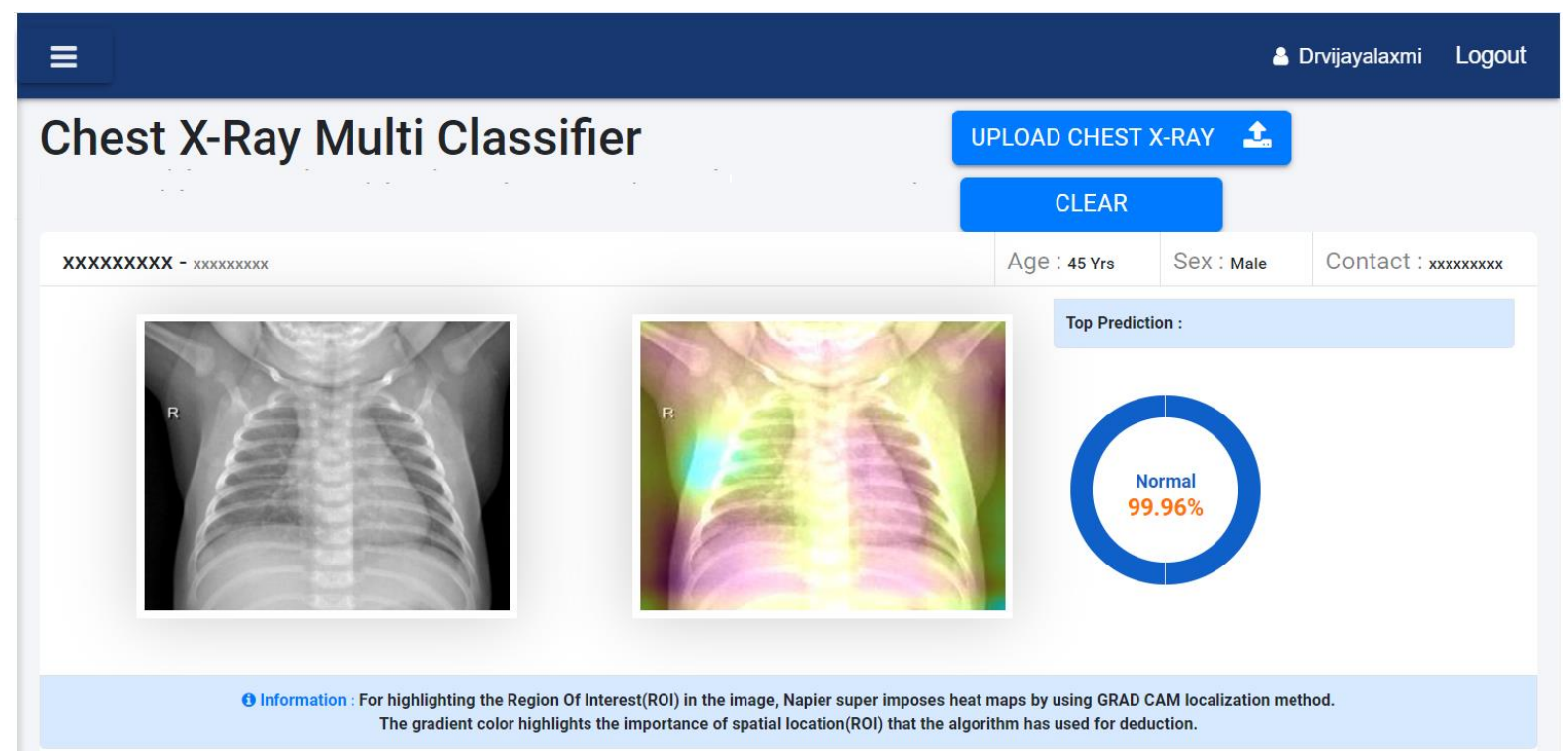

Image 1: Gradcam Image demonstrating that the model has rightly identified the image as being normal with a probability of $99.96 \%$ 


\section{DISCUSSION}

Deep learning is a machine learning method in which a complex multi-layer neural network architecture learns representations of data automatically by transforming the input information into multiple levels of abstractions [21]. CNNs are the most commonly used deep learning networks for pattern recognition tasks in images and are trained using 'training data sets' from which the network automatically learns to extract relevant features by adjusting its weights with backpropagation. In radiology, these training sets usually consist of large numbers of handlabelled images. If trained properly, these CNNs can identify features in medical images that are beyond the threshold of human detection and extract valuable new information from them [13], [20], [21].

However, deep network architectures are highly demanding in terms of the volume of data needed to train them. The sparsity and poor quality of digital medical data available for training the models was a thus major impediment to their widespread use in healthcare until as recently as 2017.

Since then, in an effort to provide sufficient training data for the research community for the development of deep learning-based algorithms, several institutions such as the $\mathrm{NIH}$ [29], the Stanford University [11], and the Massachusetts Institute of Technology [30] have released very large public datasets of annotated Chest X-rays.

The ChestX-ray14 dataset released by the NIH [29] was the first dataset to be made publicly available in 2017 and comprises 112,120 frontal-view X-ray images with fourteen disease labels derived by datamining radiology reports using Natural Language Processing (NLP) techniques. The CheXpert dataset from Stanford University containing around 224,316 images [11] and MIMIC-CXR data set from the MIT [30] containing more than 350,000 were released in 2019.

Using these data sets, several research groups have very successfully applied CNN models to detect specific problems on Chest X-rays such as such as pneumothorax [31], pneumonia [32], [33], lung nodules [34], tuberculosis [35], [36] and the presence of medical devices in the thorax. [37], [38]. Multi-label classification, where each input sample is associated with one or several labels, has also been explored and a number of publications have reported excellent performance when trained on the NIH Chestx-ray14 [39], [40], ChesXpert [11], and the MIMIC data sets [41]. A few of these algorithms have even been approved by the FDA-approved for clinical use [42].

The Napier VGG 16 CNN model compares favourably with these previously published models and has demonstrated high accuracy in the identification of abnormal images and the automated detection of 13 abnormalities on Chest x-rays. 


\section{CLINICAL APPLICATIONS}

The Napier Deep Learning CNN algorithm can be deployed in the clinical setting in at least two different ways. Firstly, as a triage tool to prioritise, reliably identify and flag abnormal images for urgent radiologist review. This would be especially useful in high-volume settings and in areas with limited reporting radiologists. Secondly, the model may also be used as a clinical decision support tool, acting as a second opinion to perform a simple back up check on the diagnosis of the physician or to direct the attention of the physicians to findings that they may have missed.

\section{LIMITATIONS}

Although our model achieved a highly accurate performance, we acknowledge that this study has some limitations.

First, the deep learning algorithm was trained and evaluated on a single open-source data set. Therefore, variance due to new or unseen patterns in the model performance may be observed when the model is exposed to new images in the clinical setting. To overcome this, we hope to re-train our model periodically using radiologist annotated, richer, larger and more diverse datasets that will be made available to us as a result of our ongoing engagement with large hospitals and radiology centres around the world.

Our training and testing dataset consisted of around 30,000 images out of the 1.2 million images in the NIH Chest X-ray data set. This may be attributed to the fact that a large number of X-rays in the NIH dataset came from only '30,000' unique patients, and nearly 50,000 of the images were labelled 'normal', making the 'effective size' of the dataset much smaller [43].

Whilst the model demonstrated excellent performance, rigorous evaluation of the model against specialists in radiology in real world clinical settings will help further validate its accuracy.

The released $\mathrm{NIH}$ dataset included corresponding metadata on patient age, gender, number of visits to the hospital and other non-image related data that were not used while training our algorithm. Integration of this metadata into the network would help the model identify any correlation between the identified labels and individual patient traits and hence increase its efficiency.

\section{CONCLUSION}

The Napier VGG 16 CNN model has demonstrated high accuracy in the automated detection of 13 abnormalities on Chest X-rays and compares favourably with previously published models. Our findings support increasing consensus that CNN based deep learning algorithms can address unmet needs in the radiology workflow and will likely be an integral part of radiology reporting in future. Large scale and in-depth prospective trials will further validate the efficacy and accuracy and ascertain the overall impact of such applications on patient care and workflow efficiency. 


\section{REFERENCES}

[1] Emea.gehealthcarepartners.com, 2021. [Online]. Available: http://emea.gehealthcarepartners.com/images/pdfs/RapidReview--Radiology-Workforce-Review-FINAL.pdf. [Accessed: 12- Sep- 2021].

[2] R.S. Bindman, D.L. Miglioretti, and E.B. Larson. "Rising use of diagnostic medical imaging in a large integrated health system," Health Affairs (Millwood). Vol. 27, No. 6, pp 1491-1502, Nov-Dec.2008.

[3] P. Putha, M. Tadepalli, B. Reddy, T. Raj, J. A. Chiramal, S. Govil, "Can artificial intelligence reliably report chest Xrays?: Radiologist validation of an algorithm trained on 2.3 million X-rays," arXiv.org, 04-Jun-2019. [Online]. Available: https://arxiv.org/abs/1807.07455. [Accessed: 12-Sep-2021].

[4] C. Qin, D. Yao, Y. Shi, and Z. Song, "Computer-aided detection in chest radiography based on Artificial Intelligence: A survey," BioMedical Engineering OnLine, vol. 17, no. 1, 2018.

[5] H. H. Pham, T. T. Le, D. Q. Tran, D. T. Ngo, and H. Q. Nguyen, "Interpreting chest X-rays via CNNs that exploit hierarchical disease dependencies and uncertainty labels," Neurocomputing, vol. 437, pp. 186-194, May 2021.

[6] R. Hirschmann, "Singapore: Registered Diagnostic Radiology Specialists," Statista, 23-Apr-2021. [Online]. Available: https://www.statista.com/statistics/605153/singapore-medical-specialists-diagnostic-radiology/. [Accessed: 12-Sep2021].

[7] L. Delrue, R. Gosselin, B. Ilsen, A.Van Landeghem, J. De Mey and P. Duyck, "Difficulties in the Interpretation of Chest Radiography", in Coche E., Ghaye B., de Mey J., Duyck P. (eds) Comparative Interpretation of CT and Standard Radiography of the Chest, $1^{\text {st }}$ ed. Heidelberg, Berlin, Springer, 2011, pp.27-49

[8] N. J. Shaw, M. Hendry, and O. B. Eden, "Inter-observer variation in interpretation of chest X-rays," Scottish Medical Journal, vol. 35, no. 5, pp. 140-141, Oct 1990.

[9] R. M. Hopstaken, T. Witbraad, J. M. A. van Engelshoven, and G. J. Dinant, "Inter-observer variation in the interpretation of chest radiographs for pneumonia in community-acquired lower respiratory tract infections," Clinical Radiology, vol. 59, no. 8, pp. 743-752, Aug 2004.

[10] B. Moifo, E. Pefura-Yone, G. Nguefack-Tsague, M. Gharingam, J. Tapouh, A. Kengne et al, "Inter-Observer Variability in the Detection and Interpretation of Chest X-Ray Anomalies in Adults in an Endemic Tuberculosis Area', Open Journal of Medical Imaging, vol. 5, no.3, pp. 143-149, Sep 2015.

[11]J. Irvin, P. Rajpurkar, M. Ko, Y. Yu, S. Ciurea-Ilcus, C. Chute, et al. "CheXpert: A Large Chest Radiograph Dataset with Uncertainty Labels and Expert Comparison", in Proceedings of the thirty-third AAAI Conference on Artificial Intelligence (AAAI-19), vol. 33, no. 01, pp. 590-597, Jul. 2019.

[12]F. Pesapane, M. Codari and F. Sardanelli, "Artificial intelligence in medical imaging: threat or opportunity? Radiologists again at the forefront of innovation in medicine", European Radiology Experimental, vol. 2, no. 1, 2018. Available: 10.1186/s41747-018-0061-6.

[13] M. Elkin, J. Macy, V. Bordaz, and W. Weymouth, "13. 'What the radiologist should know about artificial intelligencean ESR white paper. European Society of Radiology (ESR)”, Insights into Imaging, vol.10, no.44, Apr. 2019 Available https://doi.org/10.1186/s13244-019-0738-2

[14]F. Winsberg, M. Elkin, J Macy, V Bordaz, W Weymouth, "Detection of radiographic abnormalities in Mammograms by means of optical scanning and computer analysis", Radiology, vol. 89, no.2, pp 211-215, 1967.

[15]C. Kimme, B.J. O’Laughlin, J. Sklansky. "Automatic detection of suspicious abnormalities in breast radiographs”, Data Structures, Computer Graphics and Pattern Recognition. Pp.427-447, 1977.

[16] K. Doi, (2015, Mar) Chapter 1. Historical overview. In: Li Q, Nishikawa RM, editors. Computer-Aided Detection and Diagnosis in Medical Imaging. (1st ed.) CRC Press, pp. 1-17. Available: https://doi.org/10.1201/b18191

[17] H.-P. Chan, K. Doi, S. Galhotra, C. J. Vyborny, H. MacMahon, and P. M. Jokich, "Image feature analysis and computeraided diagnosis in digital Radiography. I. Automated detection of microcalcifications in mammography," Medical Physics, vol. 14, no. 4, pp. 538-548, 1987.

[18] B. J. Erickson, P. Korfiatis, Z. Akkus, and T. L. Kline, "Machine learning for medical imaging," RadioGraphics, vol. 37, no. 2, pp. 505-515, 2017.

[19]Tang, R. Tam, A. Cadrin-Chênevert, W. Guest, J. Chong, J. Barfett, et al, "Canadian Association of Radiologists white paper on artificial intelligence in radiology," Canadian Association of Radiologists Journal, vol. 69, no. 2, pp. 120-135, 2018.

[20]E. Montagnon, M. Cerny, A. Cadrin-Chênevert, V. Hamilton, T. Derennes, A. Ilinca, et al, “Deep learning workflow in radiology: A primer," Insights into Imaging, vol. 11, no. 1, 2020.

[21]H.-P. Chan, R. K. Samala, L. M. Hadjiiski, and C. Zhou, "Deep learning in medical image analysis," Advances in Experimental Medicine and Biology, vol.1213, pp. 3-21, 2020.

[22] L. Oakden-Rayner, "The rebirth of cad: How is modern ai different from the cad we know?," Rdiology: Artificial Intelligence, vol. 1, no. 3, 2019.

[23]O. Russakovsky, J. Deng, H. Su, J. Krause, S. Satheesh, S. Ma, et al, "ImageNet large Scale visual Recognition Challenge," International Journal of Computer Vision, vol. 115, no. 3, pp. 211-252, 2015.

[24]E. J. Hwang and C. M. Park, "Clinical implementation of deep learning IN Thoracic radiology: Potential applications and challenges," Korean Journal of Radiology, vol. 21, no. 5, p. 511, 2020.

[25]Krizhevsky A, Sutskever I and Hinton GE. "ImageNet classification with deep convolutional neural network", in the 26th Conference on neural information processing systems, Lake Tahoe, USA, December 3-6, 2012

[26]K. He, X. Zhang, S. Ren, and J. Sun, "Deep residual learning for image recognition," 2016 IEEE Conference on Computer Vision and Pattern Recognition (CVPR), 2016, June 27-30;Las Vegas, USA 
[27]M. Raghu, C. Zhang, J. Kleinberg and S. Bengio. "Transfusion: Understanding Transfer Learning for Medical Imaging”, 33rd Conference on Neural Information Processing Systems (NeurIPS 2019), Vancouver, Canada, 2019

[28]Zoph B, Vasudevan V, Shlens J and Le Q.V "Learning Transferable Architectures for Scalable Image Recognition," 2018 IEEE/CVF Conference on Computer Vision and Pattern Recognition, 2018, pp. 8697-8710

[29]X. Wang, Y. Peng, L. Lu, Z. Lu, M. Bagheri, and R. M. Summers, "Chestx-ray8: Hospital-scale chest X-ray database and benchmarks on weakly-supervised classification and localization of common thorax diseases," 2017 IEEE Conference on Computer Vision and Pattern Recognition (CVPR), 2017, pp. 3462-3471.

[30]E. Johnson, T. J. Pollard, S. J. Berkowitz, N. R. Greenbaum, M. P. Lungren, C.Y Deng, et al "MIMIC-CXR, a deidentified publicly available database of Chest Radiographs with free-text reports," Scientific Data, vol. 6, no. 1, 2019.

[31]G. Taylor, C. Mielke, and J. Mongan, “Automated detection of moderate and large pneumothorax on frontal chest X-rays using deep convolutional neural networks: A retrospective study," PLOS Medicine, vol. 15, no. 11, 2018.

[32]P. Rajpurkar, J. Irvin, K. Zhu, B. Yang, H. Mehta, T. Duan, D. Ding, A. Bagul, C. Langlotz, K. Shpanskaya, M. P. Lungren, and A. Y. Ng, "CheXNet: Radiologist-level pneumonia detection on chest X-rays with deep learning," arXiv.org, 25-Dec-2017. [Online]. Available: https://arxiv.org/abs/1711.05225. [Accessed: 12-Sep-2021].

[33]K. Jaiswal, P. Tiwari, S. Kumar, D. Gupta, A. Khanna, and J. J. P. C. Rodrigues, "Identifying pneumonia in chest X-rays: A deep learning approach,” Measurement, vol. 145, pp. 511-518, 2019.

[34]J. G. Nam, S. Park, E. J. Hwang, J. H. Lee, K.N. Jin, K. Y. Lim, et al "Development and validation of Deep Learning-Based automatic detection algorithm for malignant pulmonary nodules on Chest radiographs," Radiology, vol. 290, no. 1, pp. 218-228, 2019.

[35]E. J. Hwang, S. Park, K.N. Jin, J. I. Kim, S. Y. Choi, J. H. Lee, et al, "Development and validation of a Deep Learning-Based automatic detection algorithm for active pulmonary tuberculosis on Chest radiographs," Clinical Infectious Diseases, vol. 69, no. 5, pp. 739-747, 2018.

[36]P. Lakhani and B. Sundaram, "Deep learning at Chest Radiography: Automated classification of pulmonary tuberculosis by using convolutional neural networks," Radiology, vol. 284, no. 2, pp. 574-582, 2017.

[37]H. Lee, M. Mansouri, S. Tajmir, M. H. Lev, and S. Do, “A deep-learning system for fully-automated peripherally inserted central atheter (PICC) tip detection,” Journal of Digital Imaging, vol. 31, no. 4, pp. 393-402, 2017.

[38]P. Lakhani, "Deep convolutional neural networks for endotracheal tube position and X-ray image classification: challenges and opportunities," Journal of Digital Imaging, vol. 30, no. 4, pp. 460-468, 2017.

[39]P. Rajpurkar, J. Irvin, R. L. Ball, K. Zhu, B. Yang, H. Mehta, et al, "Deep learning for chest radiograph diagnosis: A Retrospective comparison of THE CheXNeXt algorithm to practicing radiologists," PLOS Medicine, vol. 15, no. $11,2018$.

[40]P. Kumar, M. Grewal, and M. M. Srivastava, "Boosted cascaded convnets for multilabel classification of thoracic diseases in chest radiographs," Lecture Notes in Computer Science, pp. 546-552, 2018.

[41]Rubin, J., Sanghavi, D., Zhao, C., Lee, K., Qadir, A. and Xu-Wilson, M., 2021. Large Scale Automated Reading of Frontal and Lateral Chest X-Rays using Dual Convolutional Neural Networks. [online] arXiv.org. Available at: <https://arxiv.org/abs/1804.07839> [Accessed 12 September 2021].

[42]The Medical Futurist. 2021. The Medical Futurist. [online] Available at: <https://medicalfuturist.com/fdaapproved-ai-based-algorithms/> [Accessed 12 September 2021].

[43]L.O. Rayner, "Quick thoughts on ChestXray14, performance claims, and clinical tasks.", Luke Oakden-Rayner, 2021. [Online]. Available: https://lukeoakdenrayner.wordpress.com/2017/11/18/quick-thoughts-on-chestxray 14performance-claims-and-clinical-tasks/. [Accessed: 12- Sep- 2021]. 
